\title{
Obesidade em idosos do Município de São Carlos, SP e sua associação com diabetes melito e dor articular
}

\author{
Obesity among older people of the City of São Carlos, SP, Brazil, and its association \\ with diabetes mellitus and joint pain
}

Estudo desenvolvido no Depto. de Fisioterapia da UFSCar Universidade Federal de São Carlos, São Carlos, SP, Brasil

1 Fisioterapeuta; mestranda no PPG-Ft - programa de PósGraduação em Fisioterapia da UFSCar

2 Prof. Dr. do Depto, de Fisioterapia da UFSCar

3 Doutoranda no PPG-Ft da UFSCar

ENDEREÇO PARA CORRESPONDÊNCIA

Thaís Rabiatti Aurichio R. Brasil 739 Rudge Ramos 09627-000 São Bernardo do Campo SP e-mail:

thaisrabiatti@yahoo.com.br

Este estudo recebeu apoio da FAPESP - Fundação de Amparo à Pesquisa do Estado de São Paulo

APRESENTAÇÃO jun. 2009

ACEITO PARA PUBLICAÇÃO abr. 2010
Thaís Rabiatti Aurichio ${ }^{1}$, José Rubens Rebelatto ${ }^{2}$, Alessandra Paiva de Castro ${ }^{3}$

Resumo: Este estudo tem por objetivo verificar a prevalência de obesidade e sua associação com diabetes melito (DM) e dores articulares na população idosa residente em São Carlos, SP. Foram avaliadas 227 idosas (média de idade 69, 6ะ6,8 anos) e 172 idosos $(69,4 \pm 6,7$ anos) quanto ao índice de massa corporal (IMC) e questionados quanto à presença de diabetes e dor articular. Os dados foram tratados estatisticamente. Os resultados mostram que $73,6 \%$ das idosas e $66,9 \%$ dos idosos apresentavam sobrepeso ou eram obesos. As médias de IMC foram maiores nos indivíduos com idade inferior a 75 anos. Entre as mulheres da amostra foi encontrada associação entre a presença de DM, dores no pé, tornozelo e joelho e a condição "obesa". No grupo masculino, a condição obeso só apresentou associação com a presença de DM. Foi pois constatada associação entre obesidade e presença de DM. Mulheres idosas apresentam maior prevalência de obesidade e associação entre dores articulares e excesso de peso corporal.

Descritores: Artralgia; Diabetes mellitus; Idoso; Obesidade

ABSTRACT: The aim of this study was to assess prevalence of obesity and its association with diabetes mellitus (DM) and joint pain among elderly dwellers in the city of São Carlos, SP, Brazil. A total of 399 aged people - 227 women (mean age 69.6 46.8 ) and 172 men (mean age 69.4 16.7 ) - were assessed as to body mass index (BMI) and were questioned whether they had diabetes and joint pain. Data were statistically analysed. Results showed that $73.6 \%$ of the women and $66.9 \%$ of the men were overweight or obese; BMI values were higher in subjects aged under 75 . Among women, associations were found between the obese condition, presence of diabetes, and foot, ankle and knee pain. In the male group, the obese condition presented association only with presence of diabetes. An association was hence found between obesity and DM. Prevalence of obesity was greater in elderly women, who also presented association between joint pain and high BMI values.

Key words: Aged; Arthralgia; Diabetes mellitus; Obesity 


\section{INTRODUÇÃO}

As modificações no perfil demográfico brasileiro revelam o aumento da população idosa no país e são acompanhadas de mudanças no aspecto epidemiológico. Atualmente há predomínio de doenças crônicas não-transmissíveis, próprias de faixas etárias mais avançadas, dentre as quais se destaca a obesidade ${ }^{1}$.

O relatório da OMS de 2002 apontou a obesidade como uma epidemia, estando no topo de uma lista dos dez riscos para a saúde humana ${ }^{2}$. Ferreira e MagaIhães ${ }^{3}$, analisando dados de indivíduos com 65 anos ou mais no Brasil, apontam prevalência de obesidade de 5\% nos homens e $18 \%$ nas mulheres. Tal distúrbio é capaz de aumentar de forma significativa a morbimortalidade por outras doenças, como hipertensão arterial, hipercolesterolemia, diabetes melito (DM), doenças cardiovasculares, doenças osteoarticulares e algumas formas de câncer ${ }^{1,3}$.

Cerca de $90 \%$ dos casos de DM tipo 2 são atribuídos ao excesso de peso ${ }^{4}$. Gomes et al. ${ }^{5}$ verificaram que a prevalência de sobrepeso e obesidade em pacientes com DM tipo 2 foi três vezes maior do que a observada na população brasileira geral. Dados do Sistema Único de Saúde revelam que o DM tipo 2 é a quinta indicação de hospitalização no Brasil e está entre as dez maiores causas de mortalidade no país ${ }^{5}$, o que evidencia a importância do diagnóstico precoce e da prevenção dessa doença, que se caracteriza como um grave problema de saúde pública.

As doenças osteoarticulares, decorrentes ou não do excesso de peso corporal, provocam limitações nas atividades físicas e na mobilidade do indivíduo idoso, merecendo atenção especial. Nos Estados Unidos a estimativa de prevalência de tais doenças é de 15\%, aumenta progressivamente com a idade e é mais elevada em mulheres idosas e obesas ${ }^{6}$. Dentre as doenças osteoarticulares a osteoartrite $(\mathrm{OA})$ representa $70 \%$ dos casos de artrite6 e acomete cerca de $16,2 \%$ da população brasileira ${ }^{7}$. Sintomas de dor, rigidez articular e crepitações estão associados à $\mathrm{OA}^{8-10}$, que apresenta como principais fatores de risco lesões prévias, obesidade e envelhecimento ${ }^{7,8,10}$.
Há uma tendência de relação entre doenças crônicas como a obesidade, o DM e as dores articulares. Assim, o objetivo deste estudo foi verificar a prevalência de obesidade e suas associações na população idosa residente em São Carlos, SP, a fim de fundamentar a adoção de estratégias preventivas específicas.

\section{METODOLOGIA}

Participaram do estudo indivíduos com 60 anos ou mais, de ambos os sexos, residentes no Município de São Carlos, SP, Brasil. A amostra foi determinada de acordo com a população do município, levando-se em consideração quotas das variáveis idade e sexo. Assim, foi composta intencionalmente por 227 idosas com média de idade de 69,6 $\pm 6,8$ anos e 172 idosos com média de idade de 69,4 46,7 anos. Foram selecionados indivíduos freqüentadores dos locais onde há maior concentração de idosos e que fossem acessíveis aos pesquisadores. Assim, a escolha dos participantes foi feita por conveniência. Foram excluídos idosos que apresentavam curativos em pés e aqueles que eram amputados em membros inferiores.

Os dados foram coletados na Universidade Aberta da Terceira Idade, na Unidade Saúde-Escola da Universidade Federal de São Carlos (UFSCar) e em duas unidades básicas de saúde do Município de São Carlos. Os participantes receberam informações sobre o estudo e assinaram o termo de consentimento livre e esclarecido. O presente estudo foi aprovado pelo Comitê de Ética em Pesquisa em Seres Humanos da UFSCar.

O peso e a altura foram mensurados por meio de uma balança analógica com estadiômetro e devidamente calibrada; o peso foi mensurado três vezes e o valor médio foi considerado. Um mesmo avaliador tomou todas as medidas de peso e altura, que foram utilizadas para o cálculo do índice de massa corporal (IMC) dos idosos. A classificação destes segundo o IMC seguiu os padrões de corte descritos pela Organização Mundial da Saúde ${ }^{11}$, sendo considerados três grupos: normal (IMC menor que $25 \mathrm{~kg} / \mathrm{m}^{2}$ ); sobrepeso (IMC maior que $25 \mathrm{~kg} / \mathrm{m}^{2} \mathrm{e}$ menor que $30 \mathrm{~kg} / \mathrm{m}^{2}$ ); e obeso (IMC superior a $30 \mathrm{~kg} / \mathrm{m}^{2}$ ).

Os participantes responderam a um questionário-entrevista aplicado por um único avaliador em toda a coleta. O questionário incluía investigação sobre a presença de diabetes e dor em coluna, quadril, joelho, tornozelo e pés. Além disso, o voluntário também era questionado sobre a presença de reumatismo.

Os dados foram analisados de forma descritiva e por meio de testes estatísticos. A avaliação das diferenças entre médias de dois grupos foi feita utilizando-se o teste $t$ de Student e as associações entre variáveis foram feitas por meio do teste do qui-quadrado. Em todos os testes o nível de significância considerado foi de 5\%.

\section{RESULTADOS}

A amostra feminina apresentou média de IMC de $28,45 \pm 5,12 \mathrm{~kg} / \mathrm{m}^{2}$, e a amostra masculina de $27 \pm 4,24 \mathrm{~kg} / \mathrm{m}^{2}$. Entre os indivíduos obesos, 24,4\% eram homens e 33,0\% eram mulheres. Em toda a amostra 18\% eram diabéticos: na amostra masculina, a prevalência de diabéticos era de $22,1 \%$, e entre as muIheres, de apenas 15,1\%.

Para identificar diferenças de IMC entre os idosos com idade inferior a 75

Tabela 1 Índice de massa corporal (IMC, média \pm desvio padrão) dos homens e mulheres segundo a faixa etária e valor de $p$ da comparação entre as faixas

\begin{tabular}{lcccc}
\hline & \multicolumn{2}{c}{ Homens $(\mathrm{n}=172)$} & \multicolumn{2}{c}{ Mulheres $(\mathrm{n}=227)$} \\
Faixa etária & IMC & $p$ & IMC & $p$ \\
\hline 60 a 74 anos & $27,11 \pm 4,23$ & & $28,89 \pm 5,32$ & \\
75 anos ou + & $26,67 \pm 4,32$ & 0,286 & $27,23 \pm 4,34$ & 0,009 \\
\hline
\end{tabular}

Tabela 2 Índice de massa corporal (IMC, média \pm desvio padrão) dos homens e mulheres e valor de $p$ da comparação entre os sexos

\begin{tabular}{lcc}
\hline Sexo & IMC & $p$ \\
\hline Homens $(n=172)$ & $27,00 \pm 4,24$ & 0,001 \\
Mulheres $(n=227)$ & $28,45 \pm 5,12$ & \\
\hline
\end{tabular}


anos e aqueles com 75 anos ou mais, foi aplicado o teste t para duas amostras (Tabela 1). O teste apontou uma diferença significativa no grupo feminino, indicando que as idosas com idade inferior a 75 anos apresentaram média de IMC maior. Entre os homens não houve diferença significativa. O teste também foi aplicado para verificar diferenças de IMC entre homens e mulheres. Os resultados, sintetizados na Tabela 2, revelam que a média de IMC das mulheres foi significativamente maior que a dos homens.

Para averiguar associações entre as condições de peso "normal", "sobrepeso" e "obeso" e outras variáveis estudadas foi aplicado o teste do qui-quadrado. No grupo feminino, foi encontrada associação entre a condição "obesa" e a presença de diabetes, de dores no pé, no tornozelo e no joelho. Entre os homens, a condição "obeso" só apresentou associação com a presença de diabetes (Tabela 3).

Tabela 3 Associação ( $\chi^{2}$ e valor de $p$ ) entre obesidade e idade, diabetes e dores articulares, por sexo

\begin{tabular}{lrc}
\hline \multicolumn{3}{c}{ Associação com obesidade } \\
\hline Mulheres & $\chi^{2}$ & $p$ \\
Faixa etária & 3,091 & 0,213 \\
Diabetes & 12,789 & $0,002^{*}$ \\
Dor nos pés & 10,874 & $0,004^{*}$ \\
Dor nos tornozelos & 9,519 & $0,009^{*}$ \\
Dor nos joelhos & 9,138 & $0,010^{*}$ \\
\hline Homens & & \\
Faixa etária & 0,133 & 0,936 \\
Diabetes & 12,710 & $0,002 *$ \\
Dor nos pés & 3,021 & 0,221 \\
Dor nos tornozelos & 2,869 & 0,238 \\
Dor nos joelhos & 2,141 & 0,343 \\
\hline
\end{tabular}

$\chi^{2}$ : teste do qui-quadrado, 1 grau de liberdade; * associação significativa com obesidade

\section{DISCUSSÃO E CONCLUSÃO}

Os resultados mostram maior prevalência da obesidade em mulheres idosas quando comparadas aos homens da mesma faixa etária. Isso já foi encontrado por diversos autores que apontam, além da predominância da obesidade no sexo feminino, uma associação desta com fatores de risco como hipertensão, diabetes e sedentarismo ${ }^{3,12,13}$. Alguns autores $^{1,12}$ verificaram que o IMC diminuiu com a idade, concordando com os achados do presente estudo.

A predisposição das mulheres para o acúmulo de gorduras e sua maior tolerância a esse excesso de gordura corporal $^{12}$ podem explicar a alta prevalência de obesidade no sexo feminino. A diminuição da obesidade com o aumento da idade sugere que pode funcionar como um fator seletivo, já que leva à maior morbimortalidade nos grupos etários mais jovens, que portanto não alcançam idades mais avançadas ${ }^{2,12}$.

A maioria dos estudos aponta para maior prevalência de diabetes no sexo feminino, visto que as mulheres são mais obesas e há uma relação entre diabetes e obesidade, verificada por diversos autores ${ }^{1,14-17}$. Em um estudo também realizado no Município de São Carlos, 22,3\% das idosas apresentavam diabetes e, entre os idosos, essa incidência era de $16,3 \%{ }^{16}$. No presente estudo as porcentagens de diabéticos foram de $14 \%$ entre as idosas e $22 \%$ entre os idosos, sendo que em ambos os grupos houve associação positiva com a obesidade. A porcentagem superior de diabetes nos homens pode ser explicada devido ao fato de a amostra masculina ter sido avaliada em sua maioria $(74,4 \%)$ em unidades básicas de saúde (o que faz pressupor que tenham maior morbidade), enquanto que apenas $27,3 \%$ das mulheres foram avaliadas nesses locais.

Experimentos utilizando estímulos somáticos verificaram que as mulheres têm menor limiar de dor para estímulos nocivos do que os homens ${ }^{18}$. Benvenuti et $a / .{ }^{19}$, que realizaram uma pesquisa epidemiológica sobre incidência de dor nos pés entre idosos, concluíram que as mulheres têm duas vezes mais chances de apresentar dor que os homens. Segundo McCarthy et al. ${ }^{20}$, as dores crônicas são comuns nos idosos, afetam mais as mulheres do que os homens e apresentam forte associação com a obesidade. Neste estudo foi verificada uma associação positiva entre obesidade e dores nos pés, tornozelos e joelhos apenas na amostra feminina. Além do menor limiar de dor, a sobrecarga sobre os membros inferiores imposta pelo excesso de peso pode ser um fator contribuinte para a maior incidência de dor nas idosas obesas.

Alguns estudos revelam associações positivas entre obesidade e a presença de $\mathrm{OA}^{6-8,21,22}$, principalmente em articulações que sustentam o peso corporal, como joelhos e tornozelos $6,9,10,22$. No presente estudo os participantes eram questionados especificamente sobre reumatismo, com especificações sobre sintomas de artrite reumatóide, não sendo sensível para os casos de OA, o que impossibilitou a análise mais detalhada da presença de OA e os demais fatores abordados no estudo. Entretanto, talvez a associação entre dores articulares e obesidade pudesse ser explicada pela presença de OA nessas idosas, o que estaria de acordo com os achados da literatura.

Este estudo permite concluir que há associação entre obesidade e a presença de DM, e que as mulheres idosas, quando comparadas aos homens idosos, apresentam maior prevalência de obesidade e associação entre dores articulares e excesso de peso corporal. 


\section{REFERÊNCIAS}

1 Marques APO, Arruda IKG, Espírito Santo ACG, Raposo MCF, Guerra MD, Sales TF. Prevalência de obesidade e fatores associados em mulheres idosas. Arq Bras Endocrinol Metab. 2005;49(3):441-8.

2 Cruz IBM, Almeida MSC, Schwanke CHA, Moriguchi EH. Prevalência de obesidade em idosos longevos e sua associação com fatores de risco e morbidades cardiovasculares. Rev Assoc Med Bras. 2004;50(2):72-7.

3 Ferreira VA, Magalhães R. Obesidade no Brasil: tendências atuais. Rev Port Saude Publica. 2006;24(2):71-81.

4 Dias JCR, Campos JADB. Aspectos epidemiológicos da obesidade e sua relação com diabetes mellitus. Nutrire. 2008;33(1):103-15.

5 Gomes MB, Gianella ND, Mendonça E, Tambascia MA, Fonseca RM, Réa RR, et al. Prevalência de sobrepeso e obesidade em pacientes com diabetes mellitus do tipo 2 no Brasil: estudo multicêntrico nacional. Arq Bras Endocrinol Metab. 2006;50(1):136-44.

6 Machado GPM, Barreto SM, Passos VMA, Lima-Costa MFF. Projeto Bambuí: prevalência de sintomas articulares crônicos em idosos. Rev Assoc Med Bras. 2004;50(4):367-72.

7 Tamegushi ST, Trelha CS, Dallaroza MSG, Cabrera M, Ribeiro TN. Capacidade funcional de idosos com osteoartrite de joelhos e quadril. Rev Espaço Saude. 2008;9(2):8-16.

8 Vasconcelos KSS, Dias JMD, Dias RC. Impacto do grau de obesidade nos sintomas e na capacidade funcional de mulheres com osteoartrite de joelhos. Fisioter Pesq. 2008;15(2):125-3.

9 Santos MTN, Freitas AE, Lamounier JA. Obesidade e osteoartrite: atualização em implicações clínicas e metabólicas. Rev Med Minas Gerais. 2008;18(4):18(4 Supl 1):S167-72.

10 Zacaron KAM, Dias JMD, Abreu NS, Dias RC. Nível de atividade física, dor e edema e suas relações com a disfunção muscular do joelho de idosos com osteoartrite. Rev Bras Fisioter. 2006;10(3):279-84.

11 World Health Organization. Obesity: preventing and managing the global epidemic (report of a WHO consultation on obesity). Genebra; 1997.
12 Cabrera MAS, Jacob Filho W. Obesidade em idosos: prevalência, distribuição e associação com hábitos e comorbidades. Arq Bras Endocrinol Metab. 2001;45(5):494-501.

13 Santos DM, Sichieri R. Índice de massa corporal e indicadores antropométricos de adiposidade em idosos. Rev Saude Publica. 2005;39(2):163-8.

14 World Health Organization. Diabetes mellitus: report of a World Health Organization Study Group. Geneva; 1985. (WHO Technical Report Series, 727).

15 Sakata S. Diabetes mellitus entre os idosos no Município de São Paulo: uma visão longitudinal [dissertação]. São Paulo: Escola de Enfermagem, USP; 2006.

16 Feliciano AB, Moraes AS, Freitas ICM. O perfil do idoso de baixa renda no município de São Carlos, São Paulo, Brasil: um estudo epidemiológico. Cad Saude Publica. 2004;20(6):1575-85.

17 Ochoa-Vigo K, Torquato MTCG, Silvério IAS. Caracterização de pessoas com diabetes em unidades de atenção primária e secundária em relação a fatores desencadeantes do pé diabético. Acta Paul Enferm. 2006;19(3):296-303.

18 Berkley KJ. Sex differences in pain. Behav Brain Sci. 1997;20:371-80.

19 Benvenuti F, Ferrucci L, Guaralnik JM, Gangemi S, Baroni A. Foot pain and disability in older persons: an epidemiologic survey. J Am Geriatr Soc. 1995;43(5):479-84.

20 McCarthy LH, Bigal ME, Katz M, Derby C, Lipton RB. Chronic pain and obesity in elderly people: results from the Einstein Aging Study. J Am Geriatr Soc. 2009;57(1):115-9.

21 Vasconcelos KSS, Dias JMD, Dias RC. Relação entre intensidade de dor e capacidade funcional em indivíduos obesos com osteoartrite de joelho. Rev Bras Fisioter. 2006;10(2):213-8.

22 Vasconcelos KSS, Dias JMD, Dias RC. Dificuldades funcionais em mulheres obesas com osteoartrite de joelhos: relação entre percepção subjetiva e desempenho motor. Fisioter Pesq. 2007;14(3):55-61. 